Alter

Revue de phénoménologie

26 | 2018

La nature

\title{
La nature chez Schopenhauer : essence et manifestation
}

Hugo Dallacosta

\section{OpenEdition}

1 Journals

Édition électronique

URL : http://journals.openedition.org/alter/564

DOI : $10.4000 /$ alter.564

ISSN : 2558-7927

Éditeur :

Association ALTER, Archives Husserl (CNRS-UMR 8547)

\section{Édition imprimée}

Date de publication : 31 décembre 2018

Pagination : $31-45$

ISBN : 978-2-9550449-4-0

ISSN : $1249-8947$

\section{Référence électronique}

Hugo Dallacosta, « La nature chez Schopenhauer : essence et manifestation », Alter [En ligne], 26 |

2018, mis en ligne le 31 décembre 2019, consulté le 22 mars 2020. URL : http://

journals.openedition.org/alter/564; DOI : https://doi.org/10.4000/alter.564

Revue Alter 


\title{
LA NATURE CHEZ SCHOPENHAUER : ESSENCE ET MANIFESTATION
}

\author{
Hugo Dallacosta
}

« Et si la nature finissait par livrer ses mystères ? » Et d'ailleurs, à qui les livrerait-elle ? Si tant est que nous en soyons les dépositaires, que pourrions-nous faire de ces mystères délivrés, nous qui cherchons la lumière de la connaissance et la clarté de l'évidence ? Ce que Goethe ne dit pas dans cette interrogation placée par Schopenhauer en exergue du Monde comme volonté et représentation, c'est justement qu'il n'y a de mystère que pour un sujet qui fait face à la nature et qui y tient une place éminemment ambiguë. Si Schopenhauer donne à la nature un tel rôle inaugural, c' est que tout questionnement philosophique se fait sur le sol de la nature ${ }^{1}$, redoublant sa manifestation effective d'une promesse de révélation de ce qu'elle n'est pas : richesse et profusion de la nature, dimension cachée de ce qu'elle manifeste. Mais le véritable mystère de la nature vient de nous, à la fois sujets faisant face à une nature qui nous semble donnée, à la fois partie de cette nature que nous cherchons à comprendre. Il y a donc toujours une expérience entrelacée de la nature, que je cherche à connaître de l'extérieur tout en m'y insérant pleinement. Et cependant, à rappeler ainsi le sujet à sa dimension naturelle, ne prend-on pas le risque de naturaliser la subjectivité, au point de contredire l'idéalisme transcendantal pourtant toujours revendiqué par Schopenhauer? À l'inverse, que faire des sciences naturelles si l'on considère l'expérience subjective de la nature comme le cœur de la démarche philosophique? Alors que Schopenhauer

\footnotetext{
1 «Ma philosophie ne parle jamais de Coucouville-les-Nuées, mais de CE monde, c'est-à-dire qu'elle est IMMANENTE et non transcendante. Elle enseigne ce qu'est le phénomène et ce qu'est la chose en soi. Mais cette dernière n'est chose en soi que RELATIVEMENT, c' est-à-dire par rapport au phénomène », lettre 280 à Julius Frauenstädt du 21 août 1852, in A. Schopenhauer, Lettres, II, trad. C. Sommer, Paris, Gallimard, 2017.
} 
semble donner le dernier mot à une reprise philosophique des sciences naturelles, il semble pourtant se tourner vers elles quand il s'agit de confirmer son intuition philosophique ${ }^{2}$.

La question de la nature exige chez Schopenhauer d'articuler ce qui est connu de la nature - ce qui relèverait, dans sa forme la plus aboutie, des sciences naturelles - avec l'essence de cette nature, ce qui lui donne sens et réalité. La difficulté, c'est toujours de penser en même temps ce qui est accessible - mais décevant du point de vue de la signification et ce qui porte le sens - mais n'est pas connaissable au sens strict car il échappe à la représentation. C'est en cela que Schopenhauer considère la nature comme le lieu d'une tension entre "essence et manifestation », sans pour autant rabattre l'une sur l'autre en les identifiant.

Une première forme prise par cette tension est la nécessité de passer d'une observation scientifique de la nature à une métaphysique de la nature. Pourtant, on a souvent relevé un apparent renversement de la philosophie schopenhauerienne, dans la seconde moitié de son œuvre : il y aurait un tournant physiologique, un retour aux sciences de l'observation menaçant ses revendications transcendantales - pourtant toujours présentes. On passerait de la nature pour un sujet, à un sujet dans la nature sans véritable transition ni cohérence. $C^{\prime}$ est un problème que Cassirer considère longuement dans le chapitre de Le problème de la connaissance dans la philosophie et la science des Temps modernes ${ }^{3}$ consacré à Schopenhauer, mais dont Schopenhauer lui-même a bien conscience. Ce qui nous amène au problème corrélatif à ce « cercle », problème de la nature considérée sous l'angle objectif. On trouve en effet plusieurs paragraphes du livre II décrivant une épopée de la nature, de la manifestation progressive de la volonté. Il apparaît que cette cosmogonie (ou physiogonie) s'organise autour d'étapes ou de strates ontologiques que Schopenhauer appelle les Idées - qualifiées par lui de "platoniciennes ». Or s'il introduit ici les Idées comme archétypes de certains phénomènes, ce n'est qu'au livre III du Monde, consacré à l'expérience esthétique, qu'il explicitera leur statut phénoménologique. À partir de là, dans quelle mesure cette conception des Idées comme corrélats d'une pure subjectivité permet-elle à Schopenhauer de reposer la question de la nature à nouveaux frais pour lui donner une dimension phénoménologique et réaffirmer le cadre transcendantal de sa philosophie?

\footnotetext{
2 En particulier dans A. Schopenhauer, De la volonté dans la nature, trad. É. Sans, Paris, PUF, 1969. ${ }^{3}$ E. Cassirer, Le problème de la connaissance dans la philosophie et la science des Temps modernes, III : Les systèmes postkantiens, trad. Collège de philosophie, Paris, Les Éditions du Cerf, 1999.
} 
Nature chez Schopenhauer : essence et manifestation

\section{La nécessité d'une métaphysique de la nature}

\section{La nature selon la science : un système de relations causales}

Schopenhauer considère la science comme la traduction simplifiée de l'intuition ordinaire, son prolongement abstrait et conceptuel dans la réflexion. Pour lui, la réflexion a le sens littéral d'une reprise : la science n'est que la sédimentation conceptuelle de ce que nous trouvons d'abord dans l'intuition. Les sciences de la nature (ou sciences empiriques, a posteriori) sont donc l'abstraction (l'idéalisation) de relations causales déjà à l'œuvre dans l'expérience ordinaire. Il ne s'agit pas vraiment d'une critique de la science mais de la délimitation de son champ d'application ou de ses ambitions. Chaque domaine de la science est délimité par le genre de relation causale qu'elle étudie ${ }^{4}$ : causes (matérielles), excitation (la vie à ses degrés fondamentaux), motifs (des actions).

Mais cette nature des sciences empiriques n'est alors qu'un système de relations causales : la théorie scientifique est décevante par rapport à l'expérience immédiate de la nature car elle en est l'appauvrissement. Par ailleurs, une science qui perdrait le lien avec l'intuition dont elle procède s'engagerait sur la voie d'un formalisme condamné par Schopenhauer :

De même que l'on part de la lumière directe du soleil pour nous tourner ensuite vers son reflet sur la lune, de même nous partons de la représentation intuitive, immédiate, évidente et garante d'elle-même pour remonter ensuite jusqu'à la réflexion, jusqu'aux concepts de la raison, abstraits, discursifs, dont la teneur tout entière ne provient que de cette connaissance intuitive et s'y rapporte ${ }^{5}$.

Une science de la nature trop abstraite est en quelque sorte ce qui se situe au plus loin de la nature elle-même, elle en est l'expérience la plus pauvre ou la forme la plus générale.

Si l'on cherche à connaître la nature depuis les sciences naturelles, il s'agit dans tous les cas de reconduire celles-ci à l'intuition qui les fonde. Autrement dit, les sciences de la nature n'ont pas d'autonomie,

\footnotetext{
${ }^{4}$ A. Schopenhauer, Le monde comme volonté et représentation, vol. II, trad. C. Sommer et al., Paris, Gallimard, 2009, chapitre 12, «Sur la doctrine de la science».

${ }^{5}$ A. Schopenhauer, Le monde comme volonté et représentation, vol. I, trad. C. Sommer et al., Paris, Gallimard, 2009, §8, p. 130 : contrairement à Kant, Schopenhauer ne considère pas les concepts comme une source de connaissance ; ils ne sont qu'une traduction abstraite de ce que l'intuition contient avec plus de richesse.
} 
et ne sont que la forme dernière, théorique et abstraite, d'une intuition généralisée et sédimentée.

Comme toutes les preuves sont des conclusions, ce n'est tout d'abord pas une preuve qu'il faut chercher pour chaque nouvelle vérité, mais une évidence immédiate. [...] Le monde de la réflexion tout entier repose sur le monde de l'intuition et y prend ses racines. Tout évidence dernière, c'est-àdire originelle, est intuitive 6 .

La science est donc la forme qui facilite une connaissance d'un certain type - connaissance relationnelle, causale, Schopenhauer dit aussi «étiologique ». Mais si l'on veut savoir ce qui, en fait de nature, a été saisi, alors on doit s'avouer insatisfait.

\section{L'inconcevable de la science et la métaphysique de la nature}

L'insatisfaction ou la déception est une des charnières ici : ce que la science nous dit de la nature a perdu le lien avec l'expérience que nous faisons au sein de cette nature. Mais alors qu'appelle-t-on encore nature ? Paradoxalement, il n'est question de la nature qu'au moment où la science qui s'y rapporte montre ses limites, au début du livre II du Monde ${ }^{7}$. Il s'agit ici d'investir l'autre face du monde et la nature entendue comme articulation entre représentation et volonté dans le cadre d'une métaphysique de la nature. Si tout concept doit être reconduit à l'intuition qui le fonde, cette représentation intuitive se fait encore dans les formes du principe de raison, sans saisir ce qui fait sa teneur et sa signification réelle. C'est ici qu'apparaît une déception, ou un échec des sciences: les sciences de la nature n'étudient que les formes (morphologie) ou les causes (étiologie).

C'est donc négativement qu'apparaît un inconcevable pour la science de la nature : ne pouvant tout saisir sous des lois, ne pouvant tout ramener à sa cause, la science est contrainte de postuler un champ qui n'est pas le sien, et qu'elle désigne comme "force de la nature », qui s'apparente à une «qualité occulte ». C'est depuis le fonctionnement des sciences naturelles qu'apparaît ce qui leur échappe. Malgré toutes les explications de la science de la nature, manque toujours la signification de ces phénomènes. Malgré la description de toutes les relations entre ce qui apparaît, reste à connaître ce qui apparaît ${ }^{8}$. Schopenhauer recourt pour décrire cet échec à l'image récurrente

\footnotetext{
${ }^{6}$ Ibid., § 14, p. $186-187$.

7 Ibid., $\$ 17$.

8 « La philosophie contemporaine du moins ne cherche nullement D'OÙ et ce EN VUE DE QUOI le monde existe, elle recherche simplement CE QU'est le monde. », ibid., § 15, p. 216.
} 
d'une forteresse que les philosophes ont toujours pris d'assaut de l'extérieur, du dehors, se heurtant à la façade, alors que Schopenhauer le premier entend creuser une voie souterraine.

\section{Le corps, "clé de déchiffrement » de la nature}

Si la nature peut sembler énigmatique, elle porte en elle sa solution. Il se trouve que par notre corps, nous connaissons une des réalités naturelles de manière intime. Le paragraphe 18 s'ouvre sur cette révélation de l'élément métaphysique à partir de l'action du corps : si je peux voir mon corps, s'il appartient à la représentation comme corps objectif (Körper), il est aussi simultanément senti, de l'intérieur, dans une expérience charnelle, comme volonté. Ce corps objectif, objet parmi les objets, est aussi corps propre (Leib), corps vécu animé d'une volonté, de pulsions, d'un élan - aucun mot de convient, car les mots sont des concepts abstraits. Je découvre donc que chaque action de mon corps, visible dans le monde comme représentation, est identiquement un acte de ma volonté, disponible à ma seule saisie. Le corps est le lieu d'une articulation entre identité matérielle - je n'ai qu'un corps - et différence formelle radicale - Körper ou Leib sont deux modes de donation.

À partir de cette articulation intime, accessible à moi seul, je peux étendre cette double dimension à la nature dans son ensemble, par analogie : de même que mon corps objectif se donne en même temps à moi comme volonté, de même les corps que je vois autour de moi doivent être, en même temps, volonté. Il en va de la signification du monde, de sa teneur sans laquelle je ne suis assuré que de ma propre existence. Je dois donc supposer que tout objet perçu est l'essence du monde rendue visible d'une certaine manière, donné dans ma représentation selon un certain mode. On passe donc d'une conception de l'objectivité comme pôle de la représentation (corrélatif de la subjectivité mondaine) à l'objectité comme résultat d'un processus de manifestation. L'objectité fait signe vers un autre essentiel qui ne se montre qu'à travers ces formes de la représentation :

La philosophie n'est rien d'autre que la compréhension [Verständnis] exacte et universelle de l'expérience elle-même, elle est l'explicitation [Auslegung] vraie du sens et de la teneur de l'expérience. Cette teneur est l'élément métaphysique, c'est-à-dire ce qui est voilé dans le phénomène et se dissimule dans ses formes, et qui est au phénomène ce que la pensée est aux mots ${ }^{9}$.

\footnotetext{
${ }^{9}$ A. Schopenhauer, Le monde comme volonté et représentation, vol. II, op. cit., chap. 17, p. 1436.
} 
La seule philosophie de la nature envisageable est donc le prolongement d'une métaphysique du corps. Dès 1816, dans ses notes manuscrites, Schopenhauer formule son «principe révolutionnaire » opposé à tout réductionnisme mécanique et scientifique : «À partir de toi tu dois comprendre la nature, pas toi à partir de la nature $»^{10}$. Autrement dit, la métaphysique de la nature schopenhauerienne est conçue comme une investigation herméneutique de la nature à partir de l'expérience du corps propre, clé de déchiffrement de la nature (ou " sésame métaphysique »).

À ces premières lectures métaphysiques de la nature semble répondre, dans une seconde période de l'œuvre de Schopenhauer, un retour aux sciences de l'observation voire un réductionnisme surprenant. Y a-t-il donc un «tournant physiologique ${ }^{11}$ par lequel Schopenhauer opérerait une naturalisation de la subjectivité, ramenant toutes les facultés transcendantales à des déterminations physiologiques?

\section{Le « cercle » schopenhauerien : monde de l'œil et œil du monde}

\section{L'objectivation de la volonté : de la nature au cerveau}

Après avoir décrit la subjectivité transcendantale et formelle au livre I, tout l'enjeu du livre II et de la métaphysique de la nature est de rendre compte d'un second mouvement de constitution de la représentation : l'expérience intime a révélé une instance réaliste et il s'agit à présent d'accompagner son déploiement dans la nature. Schopenhauer propose au livre II du Monde une seconde navigation : on passe de l'élément métaphysique, la volonté, à sa manifestation progressive à travers la nature, qui se retrouve alors dotée d'une structure métaphysique en strates. Au plus bas degré de l'objectivation, la volonté se manifeste de manière obscure, dans les phénomènes inorganiques comme la pesanteur et la résistance (règne des causes à proprement parler). C'est par complexification progressive (et par le moteur de la conflictualité) que l'on passe aux phénomènes vivants, d'abord végétaux (règne de l'excitation, comme causalité fondamentale du vivant). C'est alors qu'apparaît l'intellect, outil au service de la volonté. L'organisme animal doit se déplacer pour chercher sa nourriture, et pour cela,

\footnotetext{
10 A.Schopenhauer, Der handschriftliche Nachlaß. Band 1. Frühe Manuskripte (1804-1818), A. Hübscher (éd.), Munich, Deutscher Taschenbuch Verlag, 1985, § 621, p. 421.

11 S. Barbera, Une philosophie du conflit : études sur Schopenhauer, trad. M.-F. Merger et O. Ponton, Paris, PUF, 2004, p. 76-82.
} 
il lui faut la représentation, donc l'intellect. Dans ce second mouvement, l'intellect est une fonction tardive et secondaire que la volonté se fait au cours de sa progressive objectivation. Autrement dit, ce second mouvement semble conduire à une naturalisation de la subjectivité humaine et une dévalorisation considérable de ce qui semblait tout-puissant dans un premier temps.

La perspective adoptée est à présent celle d'un dynamisme vivant : d'une vue synchronique de la représentation on est passé à une considération diachronique de la manifestation. La nature serait l'ensemble des formes et solutions trouvées par la volonté pour s'affirmer toujours plus, se manifestant par là-même avec une acuité toujours plus grande. Schopenhauer emploie l'expression de « corporisation » pour désigner ce processus par lequel la volonté devient monde des corps. "L'organisme est la visibilité, l'objectité de la volonté individuelle", écrit Schopenhauer à plusieurs reprises ${ }^{12}$.

L'intellect est le terme de ce processus d'objectivation, outil le plus perfectionné, le plus évolué qui soit - mais outil avant tout. D'abord chez l'animal sous forme de centre nerveux central. Puis chez l'homme, par accroissement démesuré de la « pulpe cérébrale » ou de la " masse cérébrale » selon les expressions de Schopenhauer. Mais son arrivée est si tardive, que Schopenhauer le considère comme un "phénomène secondaire ", comme un produit de l'organisme, voire comme un « parasite de l'organisme » ${ }^{13}$. Autrement dit, la volonté prend corps, et à la fin de ce processus de corporisation, comme son ultime appendice, elle «se fait» un cerveau, produit l'intellect qui rend possible la représentation et jette une lumière sur le monde. Le problème est alors évident: comment un organe, une partie de la nature, dernière extrémité d'un processus naturel et causal de manifestation peut-il être en même temps l'origine de toute représentation? Comment le sujet de la connaissance peut-il ainsi être soumis à des déterminations naturelles physiologiques - et donc connu lui-même, ce dont Schopenhauer déclarait l'impossibilité ? Il semble que cette métaphysique de la nature aboutisse à une naturalisation de la subjectivité qui n'invalide pas la lecture idéaliste mais vient simplement la concurrencer. On a plusieurs formulations, chez Schopenhauer, de ce «cercle» ou " paradoxe du cerveau ${ }^{14}$ », par exemple dans Parerga et Paralipomena:

\footnotetext{
${ }^{12}$ Cf. par exemple A. Schopenhauer, Le monde comme volonté et représentation, vol. I, op. cit., § 20, p. 259; ce qui explique une certaine fascination de Schopenhauer pour la physiognomonie, science des tendances visibles de la volonté.

${ }_{13}$ A. Schopenhauer, Le monde comme volonté et représentation, vol. II, op. cit., chap. 19, p. 1463.

14 Expression d'E. Zeller, Geschichte der deutschen Philosophie seit Leibniz, Munich, R. Oldenbourg, 1873.
} 
"Si la tête est dans l'espace, cela n'empêche pas de comprendre que l'espace $n^{\prime}$ est que dans la tête $»^{15}$.

La représentation est alors le point de départ et le terme de toute connaissance. Pour Cassirer, qui examine ce problème, l'intellect est en même temps le prius et le posterius du monde : la nature est dans mon œil, mais mon œil est de la nature. À vouloir fournir une explication scientifique de la subjectivité, à vouloir trouver confirmation chez les scientifiques qu'il lit avec attention, Schopenhauer finirait par tomber d'accord avec Cabanis, pour qui la pensée est une sécrétion du cerveau (épiphénoménisme). C'est opérer un rejet absolu de toute la dimension transcendantale. Pour Cassirer ${ }^{16}$, Schopenhauer ne fournirait en vérité que l'explication empirique d'un événement qui se situe à l'intérieur du monde, l'explication matérielle ou physiologique du surgissement de la perception visuelle, ratant par là ce qu'il voulait montrer, à savoir l'émergence du monde lui-même. Bref, Schopenhauer ne proposerait finalement qu'une théorie physicaliste de la vision (donc de la connaissance).

\section{La prégnance du transcendantal}

Cependant, il semble que Schopenhauer n'a en vérité jamais l'intention de revenir sur ce qui a été développé à partir de 1813 dans la Quadruple racine et dans la première édition du Monde en 1819. Simplement, là où il développait principalement un idéalisme transcendantal, il entend à présent intégrer ses lectures scientifiques et des données positives dans ce cadre transcendantal. Même les textes les plus ambigus portent cette subordination de toute positivité à la manière dont elle se donne à un sujet.

J'entends par OBJECTIVATION l'acte de se présenter dans le monde réel des corps. Cependant, ainsi que je l'ai explicitement montré dans le livre I et dans les Compléments correspondants, l'objectivation est elle-même entièrement conditionnée par le sujet connaissant, c'est-à-dire par l'intellect et, par conséquent, en tant que telle, absolument impensable en dehors de la connaissance que nous en avons. [...] On peut aussi définir la connaissance du monde extérieur comme la CONSCIENCE DES AUTRES CHOSES par opposition à la CONSCIENCE DE SOI. [...] Ici, ma thèse est à présent celle-ci : CE QU'EST L'INTELLECT DANS LA CONSCIENCE DE SOI, C'EST-À-DIRE SUBJECTIVEMENT, SE PRÉSENTE, DANS LA CONSCIENCE DES AUTRES CHOSES, C'EST-À-DIRE OBJECTIVEMENT, COMME ÉTANT LE CERVEAU ; ET CE QU'EST LA

\footnotetext{
${ }^{15}$ A. Schopenhauer, Parerga \& Paralipomena : petits écrits philosophiques, J.-P. Jackson (éd.), Paris, Coda, 2010, «Pensées concernant l'intellect », §30, p. 442.

${ }^{16}$ E. Cassirer, Le problème de la connaissance dans la philosophie et la science des Temps modernes, III, op. cit.
} 
Nature chez Schopenhauer : essence et manifestation

VOLONTÉ DANS LA CONSCIENCE DE SOI, C'EST-À-DIRE SUBJECTIVEMENT, SE PRÉSENTE DANS LA CONSCIENCE DES AUTRES CHOSES, C'EST-À-DIRE OBJECTIVEMENT, COMME ÉTANT L'ORGANISME TOUT ENTIER ${ }^{17}$.

Il n'y a là ni mécanisme physiologique, ni réductionnisme cérébral, contrairement à ce qu'une lecture rapide pourrait laisser penser. Ce texte rappelle certes la double modalité de donation de toute chose, mais il sert surtout de transition. Schopenhauer annonce qu'il a suffisamment considéré le monde depuis la conscience de soi, et qu'il s'avance à présent dans les déterminations positives de la nature. Tout en rappelant bien que le cadre transcendantal est toujours valable et condition de toute représentation.

En toute rigueur, le processus de corporisation décrit au livre II, la longue histoire de l'objectivation de la volonté, tout cela n'a de sens que pour une conscience qui s'en fait le spectateur. Car on a souvent décrit les «formes du principe de raison » (temps, espace et causalité), mais reste à déterminer précisément le statut de la «forme fondamentale de la représentation » (Grundform), le fait d'être-représenté, le fait que tout objet est pris dans une corrélation avec un sujet qui s'y rapporte. Autrement dit, la nature entendue comme objectivation de la volonté, cela n'a de sens que pour un processus corrélatif de subjectivation. Il n'y a donc de nature que pour un sujet qui peut la saisir. Et la formule même de Schopenhauer, "conscience des autres choses ", dit cette distance de la représentation impliquant un sujet et un objet. Considérer donc que l'intellect, dans la conscience des autres choses, se réduit au cerveau, c'est dire que le cerveau est la manière dont le sujet connaissant se donne à un autre sujet connaissant. Il n'y a de manifestation de la volonté dans la nature que pour un sujet qui la contemple. Nous voyons autour de nous, en tant que nous sommes des sujets de connaissance, la volonté en général se présenter dans des organismes, et l'intellect en particulier - comme ultime rejeton d'une volonté orientée vers une volonté de connaître - se présenter comme cerveau. Mais l'expression «se présenter » implique toujours le sujet auquel cette objectivité se présente, qui s'en fait le dépositaire, et surtout l'instance constituante ${ }^{18}$.

Il serait contradictoire de considérer que la volonté se manifeste dans des formes de l'intellect (temps, espace, chaînes causales), si

\footnotetext{
${ }_{17}$ A. Schopenhauer, Le monde comme volonté et représentation, vol. II, op. cit., chap. 20, p. 1538-1539. ${ }^{18}$ Cf. ibid., chap. 22, p. 1584 : «En soi et en dehors de la représentation, le cerveau, au même titre que toute autre chose, est lui aussi une VOLONTÉ. CAR EXISTER-POUR-UN-AUTRE [Fürein-Anderes-dasein], C'EST ÊTRE-REPRÉSENTÉ [Vorgestelltwerden], ET ÊTRE-POUR-SOI [Ansichsein], C'EST VOULOIR ».
} 
l'intellect n'est en fait formé qu'à la fin de ce processus de manifestation.

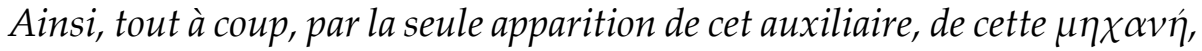
le monde comme représentation est là devant nous, pourvu de toutes ses formes : l'objet et le sujet, l'espace, le temps, la pluralité et la causalité. Le monde désormais révèle sa seconde face. Jusqu'ici simple volonté, le voilà dorénavant en même temps représentation, objet du sujet connaissant. La volonté qui, jusqu'ici, poursuivait dans l'obscurité son instinct de manière suprêmement sûre et infaillible, a, à ce degré, allumé une lumière en guise d'instrument devenu nécessaire pour annuler le désavantage né de la profusion et de la complexité de la constitution de ses phénomènes et, précisément, de ses phénomènes les plus achevés ${ }^{19}$.
\end{abstract}

Il faut donc réinterroger le statut des paragraphes 26 à 28 dans le Monde, qui retracent une épopée de la volonté se manifestant, comme une instance cosmique se déployant. Cette physiogonie du $\S 27$ n'est pas une histoire réelle de la nature, mais le récit phénoménologique décrivant l'apparition de la volonté dans une sphère du visible déjà constituée par un regard hiérarchisant, identifiant, délimitant des régions de visibilité de la volonté. À proprement parler, c'est donc moins une cosmogonie réaliste qu'une cosmologie phénoménologique.

Pour mieux comprendre cela, pour rendre cette lecture plus légitime et cohérente, il faut s'appuyer sur le concept d'Idées, introduit ici au livre II par Schopenhauer alors qu' au premier abord, elles semblent n' avoir rien à y faire, puisqu'elles sont décrites, dans le titre du livre III, comme «l'objet de l'art». Repenser le statut des Idées chez Schopenhauer permet de reposer à nouveaux frais la question de la nature, mais aussi d'élargir considérablement le champ problématique généralement associé au livre III - l'art et l'expérience esthétique.

\title{
III. Les Idées dans la nature : la structure phénoménologique de la manifestation
}

\section{L'introduction des Idées}

Les Idées apparaissent au $\S 25$ du Monde comme volonté et représentation, alors qu'il est question de l'objectivation de la volonté en nature et qu'il faut assurer l'articulation entre la volonté, totalement étrangère aux formes spatio-temporelles de la représentation, et la pluralité des

${ }_{19}$ A. Schopenhauer, Le monde comme volonté et représentation, vol. I, op. cit., § 27, p. 331-332. 
choses dans lesquelles elle se manifeste, donc la nature dans son ensemble. Les Idées sont alors conçues comme des degrés caractéristiques de visibilité de la volonté, entre la profusion des corps particuliers et l'indivisible unité de la volonté. Schopenhauer renvoie au livre suivant pour leur examen approfondi, mais recourt déjà à ces « différents degrés d'objectivation de la volonté », "formes éternelles des choses »: elles ne sont donc soumises ni au temps ni à l'espace, qui relèvent du principe d'individuation ${ }^{20}$. En somme, les Idées permettent à la volonté de s'objectiver avec la plus grande clarté, de connaître une multiplicité de strates plutôt qu'une multiplicité disparate et profuse. Là encore, c'est la dimension herméneutique qui justifie ces formes intermédiaires : grâce aux Idées, la nature, sa hiérarchie ou sa structure sont plus lisibles. Dès l'introduction du concept, Schopenhauer précise qu'elles ne sont en rien des "productions abstraites de la raison dogmatisant sur le mode scolastique ${ }^{21}$, mais qu'elles ont le sens que Platon leur accorde - à condition, en vérité, que l'on considère les Idées ou Formes chez Platon dans leur lien avec la noèse qui les saisit, donc en fait dans une lecture plus plotinienne que platonicienne.

Je comprends donc sous le mot IDÉE ce DEGRÉ D'OBJECTIVATION DE LA VOLONTÉ, fixe et déterminé, dans la mesure où la volonté est la chose en soi et, par suite, étrangère à la pluralité, tandis que ces degrés se rapportent aux choses particulières comme leurs formes éternelles ou leurs prototypes ${ }^{22}$.

Autrement dit, à partir du moment où elles abandonnent la stricte unité de la volonté, elles sont du côté de la représentation. Ce qui signifie aussi que la représentation peut avoir plusieurs formes, y compris hors du principe de raison (conscience des autres choses, conscience de soi, expérience esthétique). Les Idées sont donc les formes les plus claires de la manifestation de l'essence, sans être recouvertes par trop de déterminations particulières.

La philosophie, en revanche [à la différence de l'étiologie et de la morphologie], considère dans tout, et donc aussi dans la nature, seulement le général ; ici, elle prend pour objet les forces originelles et elle reconnaît dans cellesci ces différents degrés de l'objectivation de la volonté, qui est l'essence intime et l'en-soi de ce monde qu'elle déclare, abstraction faite de cet en-soi, n'être que la représentation du sujet ${ }^{23}$.

\footnotetext{
${ }^{20}$ Aux formes transcendantales du principe de raison correspondent les déterminations effectives du principe d'individuation. Les deux sont corrélatives.

${ }_{21}$ A. Schopenhauer, Le monde comme volonté et représentation, vol. I, op. cit., § 25, p. 295-296.

22 Ibid., p. 296.

${ }^{23} \mathrm{Ibid}$., § 27, p. 315.
} 
La volonté ne peut se manifester que dans une représentation, ici une représentation bien particulière, donc pour un sujet qui examine la nature et décèle en elle des degrés de visibilité de l'essence dynamique, la volonté. Schopenhauer décrit longuement la conflictualité à l'œuvre dans une sorte d' " assimilation dominante ", qui permet d'expliquer à la fois les différences évidentes entre les différentes strates de la nature, de l'inorganique jusqu'à l'être humain, à la fois l'unité analogique - du fait d'une essence commune - entre tous ces phénomènes $^{24}$. Et là encore, ce sont les Idées qui jouent le rôle de liaison, tenant ensemble l'unité indissoluble de la volonté et la multiplicité de la représentation ordinaire grâce à des formes présentes à tous les degrés de la nature (la polarité, la conflictualité...). Schopenhauer est bien conscient, dans ces moments de «l'obscurité inhérente à ces pensées $»^{25}$. Pour y voir plus clair, il faut suivre ses propres indications dans la préface, à savoir lire deux fois l'ouvrage pour connaître par avance les acquis du livre suivant.

\section{L'expérience esthétique : Idée et corrélation}

Si l'on a pu retenir que le livre III examine la question de l'art, il considère surtout, et avant tout la représentation pure, la représentation hors des formes mondaines du principe de raison, dans ce qu'elle a de plus essentiel. Le livre III décrit certes les différentes formes d'art, mais entendues comme reproductions techniques d'un certain type d'intuition. Ou plutôt, chaque forme d'art correspond à l'intuition des différentes Idées - de l'architecture, fondamentale, jusqu'à la musique, la plus détachée des déterminations matérielles. L'essentiel de ce livre se trouve dans les passages consacrés à l'expérience esthétique, qui recouvre en vérité bien plus que l'expérience devant l'œuvre d'art. L'œuvre d'art est l'occasion privilégiée de cette intuition du monde qui peut également avoir lieu face à la nature.

La description de la saisie intuitive des Idées permet à Schopenhauer de rappeler un élément fondamental et pourtant souvent peu remarqué : l'expérience esthétique, puisqu'elle saisit les Idées qui ne sont pas soumises aux formes du principe de raison, ne laisse subsister de la représentation que sa Grundform, la relation entre un sujet et un objet. Or la qualité de pure objectivité de l'Idée implique corrélativement une modification du sujet individuel en pure subjectivité : il

\footnotetext{
${ }^{24}$ Cf. ibid., p. 322-324.

${ }^{25} \mathrm{Ibid}$., p. 323 ; et Schopenhauer poursuit sur l'attitude nécessairement active de son lecteur: «seulement, je vois bien que je dois compter sur la réflexion propre du lecteur si je ne veux pas rester incompris ou mal compris ».
} 
passe d'un sujet mondain, déterminé dans le temps, l'espace et la causalité, à une pure conscience capable de saisir les degrés de visibilité de la volonté. Autrement dit, le geste philosophique du début de ce livre déborde très largement les limites de l'art. L'œuvre est le déclencheur de cette altération philosophique du sujet et de l'objet, et permet de donner à voir, dans la représentation, la corrélation entre pur sujet et Idée de la manière la plus claire qui soit.

Si nous nous élevons de la connaissance des choses singulières aux Idées, cela ne saurait s'accomplir qu'à condition que se produise une altération dans le sujet ; c'est en vertu de cette altération, correspondant, analogiquement, à ce grand changement du mode de l'objet dans son intégralité, que le sujet, dans la mesure où il connaît une Idée, n'est plus un individu².

Schopenhauer décrit ici les caractères d'une corrélation entre la structure subjective et les déterminations de l'objet, l'une et les autres n'étant qu'une seule et même chose. Cette corrélation est renforcée par les images de «pôles » subjectif et objectif ou de "polarité » comme forme fondamentale auxquelles Schopenhauer renvoie régulièrement $^{27}$. Pour Schopenhauer, il y a là, enfin, un accès authentique au monde, et non seulement aux relations entre les choses. Le sujet de l'expérience esthétique, devient «pur sujet, clair miroir de l'objet». Alors que Schopenhauer nous annonçait un passage à l'œuvre d'art, il est surtout question de la nature dans ces paragraphes 33 à 35. L'altération du sujet, le passage à une représentation pure et adéquate, si elle est facilitée par l'art, est avant tout la manière adéquate de considérer la nature : structurée par des Idées, comme degrés de visibilité, corrélats objectifs d'une subjectivité altérée et purifiée. C'est en ce sens que Schopenhauer écrit :

Celui donc qui, comme on l'a dit, s'est immergé et perdu dans l'intuition de la nature, au point de ne plus exister que comme pur sujet connaissant, devient conscient, de façon intime et immédiate, qu'à ce titre il est la condition, c'est-à-dire le support du monde et de toute existence objective, puisque celle-ci se présente désormais comme dépendante de la sienne ${ }^{28}$.

$* * *$

\footnotetext{
26 Ibid., § 33, p. 371.

${ }^{27} \mathrm{Ibid}$., $\$ 26$ notamment. Les similitudes avec la réduction transcendantale husserlienne sont ici réelles. Le lien entre réduction et expérience esthétique n'est cependant mentionné explicitement qu'une fois par Husserl, dans la lettre à Hofmannsthal du 12 janvier 1907, traduite dans E. Escoubas (éd.), Art et phénoménologie, Bruxelles, La Part de l’œil, 1991.

${ }^{28}$ A. Schopenhauer, Le monde comme volonté et représentation, vol. I, op. cit., § 34, p. 378.
} 
L'hypothèse selon laquelle c'est le statut reconsidéré des Idées et leur saisie intuitive - expérience esthétique - qui permettent de comprendre adéquatement la philosophie de la nature développée au livre précédent semble donc confirmée. Il s'agit d'opérer une lecture circulaire, ou par reprise conceptuelle : la saisie intuitive des Idées implique une altération du sujet dans son rapport à la nature. L'épopée physiogonique que l'on trouve aux paragraphes 26 à 28, lieu de la conflictualité entre des Idées conçues dans un premier temps comme strates ontologiques, a vu son statut modifié. Ce n'est qu'un pur sujet de la connaissance qui peut saisir la nature dans sa structure essentielle - dans sa stratification phénoménologique. Autrement dit, il n'y a jamais de description de type évolutionniste ou morphogénétique chez Schopenhauer, mais ces paragraphes sont bien plutôt un récit phénoménologique opéré depuis une subjectivité qui s'absorbe dans la contemplation adéquate de la nature.

Les Idées sont bien des degrés de visibilité : d'une part, elles sont ramenées à l'unité sous-jacente de la volonté, c'est-à-dire que c'est une seule et même chose qui se montre. D'autre part, elles relèvent de la manifestation, donc d'une distance même minime entre un sujet et un objet, pur sujet disponible à la saisie de cette pure objectivité du monde. Si donc on veut parler de strates ontologiques, ou même de "régions ontologiques », il faut se conformer à l'usage phénoménologique de ces expressions. À savoir que les différentes régions de l'être impliquent une certaine manière de se donner à une conscience. Autrement dit, la délimitation des régions se fait selon les critères de la donation. Avec son vocabulaire parfois incertain, c'est précisément ce que Schopenhauer décrit dans ces passages : les Idées sont à chaque fois une manière qu'a la volonté de se montrer. Or toute manifestation implique une subjectivité corrélative, une certaine disposition du sujet à son égard. Penser la nature comme une stratification des visibilités de la volonté, c'est donc la penser dans le cadre transcendantal de la corrélation. Ce qui implique alors de nuancer une conception du sujet et de l'objet - ou de l'homme et de la nature - comme opposition ou séparation insolubles. Il s'agit bien plutôt de les reconduire à un fond commun, à une vie commune dont ils surgissent comme des pôles indissociables. En ce sens, on écarte l'idée d'une subjectivité pleinement naturalisée, qui n'aurait plus de sens, sauf à nier la subjectivité elle-même. Toute dimension naturelle est reconduite à une conscience qui s'en saisit, à laquelle elle se donne, et le cadre transcendantal de l'idéalisme schopenhauerien se voit réaffirmé.

Schopenhauer est conduit à la nécessité d'une forme de réduction, d'expérience suspendue et purifiée du monde. En ce sens, il fait 
apparaître la nature non pas dans la seule représentation mondaine, confuse ; non pas la nature selon la science, appauvrie par le processus d'abstraction conceptuelle; non pas, enfin, ramenée toujours à une essence qui se déroberait. Mais il élabore une philosophie de la nature complète sollicitant le sujet, exigeant de lui qu'il modifie ses dispositions à l'égard de la nature car il est partie prenante de celle-ci pour des raisons phénoménologiques. Car il n'est pas un sujet isolé ou toutpuissant, il n'est pas la liberté opposée au déterminisme naturel. Il est de la nature, dans la nature, et celle-ci exige de lui, pour être saisie dans son apparaître authentique, dans sa structure phénoménologique véritable, un ajustement de la perception et de la représentation. Il n'y a donc pas nécessairement une exigence axiologique ni même éthique en provenance de la nature pour Schopenhauer, mais la conscience d'un destin commun de l'un et l'autre, d'une intégration véritable de la manière dont on considère la nature au sein de notre nature propre. 